\title{
Inter-subject Modelling of Liver Deformation During Radiation Therapy
}

\author{
M. von Siebenthal ${ }^{1}$, G. Székely ${ }^{1}$, A. Lomax $^{2}$, and Ph. Cattin ${ }^{1}$ \\ ${ }^{1}$ Computer Vision Laboratory, ETH Zurich, 8092 Zurich, Switzerland \\ \{mvonsieb, szekely, cattin\}@vision.ee.ethz.ch \\ ${ }^{2}$ Paul Scherrer Institut, 5232 Villigen PSI, Switzerland
}

\begin{abstract}
This paper presents a statistical model of the liver deformation that occurs in addition to the quasi-periodic respiratory motion. Having an elastic but still compact model of this variability is an important step towards reliable targeting in radiation therapy. To build this model, the deformation of the liver at exhalation was determined for 12 volunteers over roughly one hour using 4DMRI and subsequent non-rigid registration. The correspondence between subjects was established based on mechanically relevant landmarks on the liver surface. Leave-one-out experiments were performed to evaluate the accuracy in predicting the liver deformation from partial information, such as a point tracked by ultrasound imaging. Already predictions from a single point strongly reduced the localisation errors, whilst the method is robust with respect to the exact choice of the measured predictor.
\end{abstract}

\section{Introduction}

The variability in shape and position of abdominal organs is an important issue in image acquisition, segmentation, patient setup and treatment. In the liver, the respiratory motion can lead to displacements of several centimetres [1]. Within a few minutes, a high reproducibility of the respiration was reported [2]. However, over larger intervals of $20 \mathrm{~min}$ or more, deformations of more than one centimetre were observed in addition to the quasi-periodic respiratory motion [3]. In radiation therapy such deformations lead to large uncertainties in target localisation.

One approach to improve targeting during treatment is to use deformation models as prior knowledge [4]. For the liver, models have been proposed that capture the subject specific quasi-periodic motion [5]. In contrast, we propose an inter-subject model, which is built from a training set of livers and can be applied to a previously unseen liver. Inter-subject models have been proposed for the cardiac shape and motion, which is close to periodic [6]. In the liver, the quasi-periodic component of motion can be handled to a large degree by breathhold techniques or respiratory gating. Thus, the aim of this paper was to model the systematic motion and deformation of the liver, which occurs in addition to the quasi-periodic motion and can be considered as superimposed.

One direct application of this model is to predict the deformation of the entire liver from partial information. As a result, the displacement of a liver 
tumour could be predicted from the displacement of vessel branchings that are tracked by ultrasound imaging, even though the tumour itself may not be visible in this modality. A deformable organ model offers the flexibility of non-rigid transformations between the planning and the treatment scene and should at the same time restrict the deformation to a tractable number of parameters.

\section{Methods}

\subsection{Data Acquisition}

To capture the intra-fraction deformation of the liver, 4DMRI sequences 3 were acquired from 12 healthy volunteers ( 6 female, 6 male, average age 31, range 17-75) after written consent was obtained. This produced time-resolved MR volumes consisting of 25-30 slices with a thickness of $4 \mathrm{~mm}$ and an in-plane resolution of $192 \times 192$ pixels and $1.8 \times 1.8 \mathrm{~mm}^{2}$. MR volumes with a temporal resolution of 290-410 ms were reconstructed, which produced 3300-6700 volumes during acquisition sessions of 40-75 min. In the following, we will only consider the variability of the liver at exhalation. Therefore, the volume with the most superior liver position was selected from each breathing cycle, yielding 220-850 exhalation volumes per volunteer.

The deformation of the liver at exhalation was determined for each volunteer by intensity-based non-rigid registration 37] between the reconstructed volumes. The resulting deformations describe the variability of the exhalation position at each point within the liver over time. Figure 1 illustrates how the exhalation position of discrete points varied over an acquisition session of $51 \mathrm{~min}$. In addition to a small variation from cycle to cycle we observe large systematic displacements of more than $15 \mathrm{~mm}$. Such deviations can be caused by drifts in the exhalation level, peristalsis, muscle distension or moving gases.
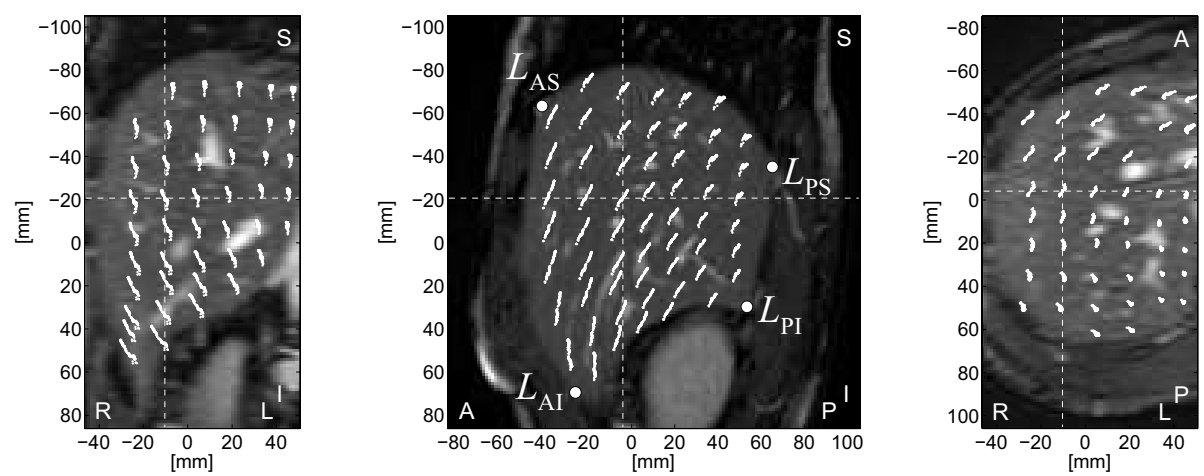

Fig. 1. Example point clouds in subject 1 showing a drift of the exhalation position in superior direction over $51 \mathrm{~min}$ 


\section{$2.2 \quad$ Inter-subject Correspondence}

A key component for building a statistical model that covers different subjects is the inter-subject correspondence. This raises the question, with respect to which criterion two points should correspond. For example, in resective liver surgery correspondence should be based on the vascularisation. However, for modelling deformation in the abdomen, the goal is to find corresponding points that move comparably. We assume that for mechanical considerations the most relevant property of a point in the liver is its location relative to the liver surface, for example its distance from the diaphragm. We thus propose a scheme to establish correspondence based on landmarks on the liver surface.

In a preparatory step, the liver was segmented manually in one exhalation volume per subject. This produced fine triangular surface meshes with several thousand triangles but without correspondence between subjects. These surfaces were then remeshed with an approach similar to 8 in order to establish correspondence. For this purpose, mechanically relevant landmarks were identified. Figure 1 shows four landmarks $L_{\mathrm{AI}}, L_{\mathrm{AS}}, L_{\mathrm{PI}}, L_{\mathrm{PS}}$, where the indices indicate the location (anterior, posterior, superior, inferior). These points mark the delineations between the superior surface in contact with lung, the anterior and the posterior areas, which slide along the abdominal wall, and the inferior surface. The four landmarks were labelled manually in each sagittal slice from the inferior vena cava (IVC) to the inferior tip of the liver and were connected by B-splines. A prototype of the right liver lobe consisting of 46 triangles was then aligned to the fine mesh of each specific liver as illustrated in Fig. 20, such that its four edges coincided with the marked delineations. In medio-lateral direction, the vertices of the prototype were regularly distributed along the landmark splines. The coarse prototype was then gradually refined to fit the fine surface mesh. In each refinement step, the triangles of the prototype were regularly subdivided into four smaller triangles and the newly generated points were projected onto the surface mesh. Figure $2 \mathrm{~b}$ shows how a new point $P_{\mathrm{ab}}^{\prime}$ was projected along the normal $n$.

Three refinement steps were performed for each of the 12 livers (Fig. 2r c). Based on the resulting correspondences on the liver surface, a grid of corresponding points within the liver was defined. A regular grid of 290 points with $15 \mathrm{~mm}$ resolution was placed in the average liver shape and then transformed to each subject specific liver. Therefore, a Delaunay tetrahedrisation of the average liver was produced. The position of each point in the average liver is defined by the barycentric coordinates that give its position within a tetrahedron. Assuming the same tetrahedrisation in each subject specific liver, the grid point can be transformed to these livers by placing it at the same barycentric coordinates. The resulting points in each liver, which correspond to the regular grid of points in the average liver, will be further used for statistical modelling.

\subsection{Statistical Modelling}

We propose a model of the liver deformation that occurs during a treatment session in addition to the quasi-periodic breathing. The model captures the drift 

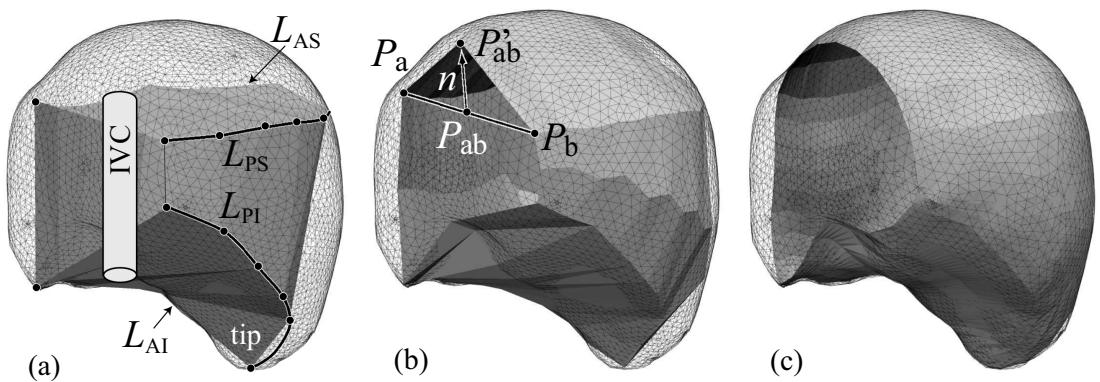

Fig. 2. (a) Coarse mesh prototype aligned with respect to manually identified delineations on the liver surface. (b) Refined mesh after correspondence preserving subdivision and projection. (c) Resulting mesh after three refinement steps.

of the exhalation position in each corresponding point. It should be noted that our aim was not to model the liver anatomy, which would describe the intersubject variation of the position of the corresponding points. Instead, we describe the drift of each corresponding point in a point specific coordinate system, which is placed at the corresponding position in each subject. The axes of the point specific coordinate systems are chosen to reflect the orientation of the liver in the body. The left-right axis is perpendicular to the medial plane of the body and the superior-inferior axis points along the posterior abdominal wall.

To avoid a bias towards one specific dataset, an equal number of 200 sample exhalation positions was taken from each dataset, equally distributed over the duration of the acquisition session. From this data, a point distribution model was built, with $M=290$ corresponding points and $N=12 \times 200$ samples of each point. Each instance $i$ of the liver was specified by a $3 M$-dimensional vector $p_{i}$ containing the position of each point. Principal component analysis was applied to determine the eigenvectors of the covariance matrix, i.e. the main modes of variation [9. We assume that the matrix $U$ is composed of the principal components as column vectors and that the diagonal matrix $\Lambda$ contains the eigenvalues. With the mean shape vector $\bar{p}$, a specific instance can be defined as $p_{i}=\bar{p}+U b_{i}$, where $b_{i}$ are the coefficients of the linear combination of eigenvectors.

\subsection{Drift Prediction}

The derived model can be used to predict the displacement of all considered points from a few measurements. Assuming that the displacement of a point $j$ from its mean position is known, the most probable deformation that fulfils the constraint imposed by this fixed point is the one with minimum Mahalanobis distance from the mean shape. Based on the submatrix $U_{j}$, which contains only those rows of $U$ that correspond to point $j$, we can calculate basis vectors $r_{x_{j}}, r_{y_{j}}, r_{z_{j}}$ that cause a unit translation of point $j$ and have minimal Mahalanobis length [9]. With these basis vectors and the known point displacement $\left[\Delta x_{j}, \Delta y_{j}, \Delta z_{j}\right]^{T}$, the most probable instance $\check{p}$ is determined as 


$$
R_{j}=\left[r_{x_{j}} r_{y_{j}} r_{z_{j}}\right]=\Lambda U_{j}^{T}\left[U_{j} \Lambda U_{j}^{T}\right]^{-1}, \quad \check{p}=\bar{p}+U R_{j}\left[\begin{array}{c}
\Delta x_{j} \\
\Delta y_{j} \\
\Delta z_{j}
\end{array}\right] .
$$

If we assume that any point in the liver could be used as a predictor, we would choose the point $P_{\text {opt }}$ that reduces the variance of the model as much as possible. As further elaborated in [9], the basis vectors $R_{j}$ can be used to remove the variance of point $j$ from the statistical model by subtracting $R_{j}$, weighted by the example specific displacements $\left[\Delta x_{j}, \Delta y_{j}, \Delta z_{j}\right]_{i}^{T}$, from the parametric representation $b_{i}$ of each shape instance $i$.

$$
\check{b}_{i}=b_{i}-R_{j}\left[\begin{array}{c}
\Delta x_{j} \\
\Delta y_{j} \\
\Delta z_{j}
\end{array}\right]_{i}=b_{i}-R_{j} U_{j} b_{i}, \quad \forall i \in\{1, \ldots, N\}
$$

To determine by how much the knowledge of point $j$ has reduced the variability of the model, the variance of the coefficients $\breve{b}_{i}$ after model reduction is compared to the variance of $b_{i}$ before reduction. The point with maximum reduction potential, $P_{\mathrm{opt}}$, is selected among all considered points and used as a predictor for the deformation of the entire liver.

To evaluate the predictive power of the model, leave-one-out experiments were performed. Models built from 11 of the 12 livers were used to predict the displacement of all points in the left-out liver following (11). In a practical setup it might not be possible to measure $P_{\mathrm{opt}}$, for example due to the lack of trackable structures. For this reason, all 290 corresponding points were tested for their capability to predict the deformation of the left-out liver.

\section{Results}

The compactness of the derived model is characterised by the cumulative percentage of variance covered by the first few principal components. The cumulative variance was calculated from the eigenvalues $\Lambda$ of a model containing all $12 \times 200$ sample exhalation positions.

\begin{tabular}{|c|c|c|c|c|c|c|}
\hline components & 1 & 2 & 3 & 4 & 5 & 6 \\
\hline cumulative variance [\%] & 78 & 88 & 91 & 94 & 96 & 97 \\
\hline
\end{tabular}

Although the model describes the distribution of 290 points over 2400 sample deformations, it is compact and covers $91 \%$ of the full variance with 3 principal components. This shows that there are typical modes of deformation during treatment. Note that this model of intra-fraction deformation is not subject specific and has to be distinguished from a model of anatomical inter-subject variation, where with only 12 sample shapes a compact model would be less surprising.

Table 1 shows the extent of deformation observed in each subject over the acquisition sessions of 40-75 min. Whilst the mean displacement of all considered points is $5 \mathrm{~mm}$ or larger in 5 of 12 subjects, the maximum displacement is $5 \mathrm{~mm}$ or 
Table 1. Euclidean displacements during complete acquisition sessions; average \pm standard deviation and (maximum) over the right liver lobe. The prediction errors are given for three cases: (A) prediction from $P_{\mathrm{opt}}(\mathrm{B})$ worst prediction from one of the six neighbours of $P_{\text {opt }}(\mathrm{C})$ prediction from the three points with maximum reduction potential according to the model.

\begin{tabular}{|c|l|l|l|ll|}
\hline subject & $\begin{array}{c}\text { deformation } \\
{[\mathrm{mm}]}\end{array}$ & $\begin{array}{c}\text { error A }[\mathrm{mm}] \\
P_{\text {opt }} \text { known }\end{array}$ & $\begin{array}{c}\text { error B }[\mathrm{mm}] \\
1 \text { point known }\end{array}$ & \multicolumn{2}{|c|}{$\begin{array}{c}\text { error C } \\
3 \text { points known }\end{array}$} \\
\hline \hline 1 & $6.5 \pm 3.4(18.8)$ & $2.3 \pm 1.3(7.7)$ & $2.6 \pm 1.5(8.7)$ & $1.9 \pm 1.2$ & $(5.8)$ \\
2 & $3.4 \pm 1.7(7.1)$ & $1.6 \pm 0.7(3.2)$ & $1.8 \pm 0.7(3.5)$ & $1.1 \pm 0.5$ & $(2.7)$ \\
3 & $3.2 \pm 1.7(8.4)$ & $1.8 \pm 0.9(5.1)$ & $2.3 \pm 0.9(6.5)$ & $1.4 \pm 0.7$ & $(4.0)$ \\
4 & $6.1 \pm 2.9(14.8)$ & $2.0 \pm 0.7(3.3)$ & $2.2 \pm 0.8(3.9)$ & $1.4 \pm 0.5$ & $(2.6)$ \\
\hline 5 & $5.5 \pm 2.2(10.2)$ & $1.5 \pm 0.4(3.2)$ & $1.5 \pm 0.5(3.8)$ & $1.0 \pm 0.3$ & $(2.2)$ \\
6 & $3.9 \pm 0.9(5.7)$ & $1.6 \pm 0.5(3.0)$ & $1.6 \pm 0.5(3.1)$ & $0.9 \pm 0.4$ & $(1.9)$ \\
7 & $3.1 \pm 1.5(7.0)$ & $0.9 \pm 0.4(2.0)$ & $1.0 \pm 0.4(2.0)$ & $0.7 \pm 0.4$ & $(1.8)$ \\
8 & $5.5 \pm 3.0(12.1)$ & $1.6 \pm 1.0(5.0)$ & $2.0 \pm 1.2(5.8)$ & $1.2 \pm 0.6$ & $(3.1)$ \\
\hline 9 & $5.0 \pm 1.9(10.1)$ & $1.3 \pm 0.6(3.8)$ & $1.4 \pm 0.7(4.0)$ & $0.9 \pm 0.5$ & $(2.7)$ \\
10 & $2.4 \pm 1.0(5.0)$ & $0.6 \pm 0.2(1.4)$ & $0.6 \pm 0.2(1.5)$ & $0.5 \pm 0.2$ & $(1.5)$ \\
11 & $3.1 \pm 1.4(6.0)$ & $1.5 \pm 0.6(3.0)$ & $1.6 \pm 0.7(3.3)$ & $0.9 \pm 0.4$ & $(2.4)$ \\
12 & $4.2 \pm 2.1(9.4)$ & $1.7 \pm 0.8(4.2)$ & $2.0 \pm 0.8(4.4)$ & $1.3 \pm 0.6$ & $(3.0)$ \\
\hline
\end{tabular}

larger in all subjects and ranges up to $18.8 \mathrm{~mm}$. This confirms that the considered deformations are an important source of localisation uncertainties.

Figure 3 depicts the results of the leave-one-out experiment for subject 1 . The drift of each corresponding point over the acquisition session of $51 \mathrm{~min}$, was predicted from a single point, for which we assume that the drift can be measured exactly. Each corresponding point was tested by using it as a predictor for the entire deformation. The resulting average error for each predictor is shown as the size of the marker at the respective position. Most points show a similar predictive power, which illustrates that the method is robust with respect to the choice of the predictor. However, we can identify two areas that are less suitable as predictors. First, this is the medial area toward the inferior vena cava, where the influence of the beating heart is largest, and second, points in the tip of the liver lobe show lower predictive power. These points are directly influenced by intestinal motion, which is in some cases poorly correlated to the deformation in the rest of the liver.

Table 1 shows the results for all 12 leave-one-out experiments and three different kinds of available partial information.

Case $A$ : The prediction of the liver deformation based on the point $P_{\mathrm{opt}}$, which has the maximum potential for variance reduction according to the model, leads to small average prediction errors ranging from $0.6 \mathrm{~mm}$ to $2.3 \mathrm{~mm}$. Also the maximum prediction error is clearly smaller than the full deformation in all subjects. For subject 1, the location of $P_{\text {opt }}$ is shown in Fig. 3. Not surprisingly, this point lies near the centre of the considered region. In subject 3 , the deformation is successfully predicted with a mean residual error of $1.8 \mathrm{~mm}$, but the maximum error is only reduced from $8.4 \mathrm{~mm}$ to $5.1 \mathrm{~mm}$. This is possible because the 

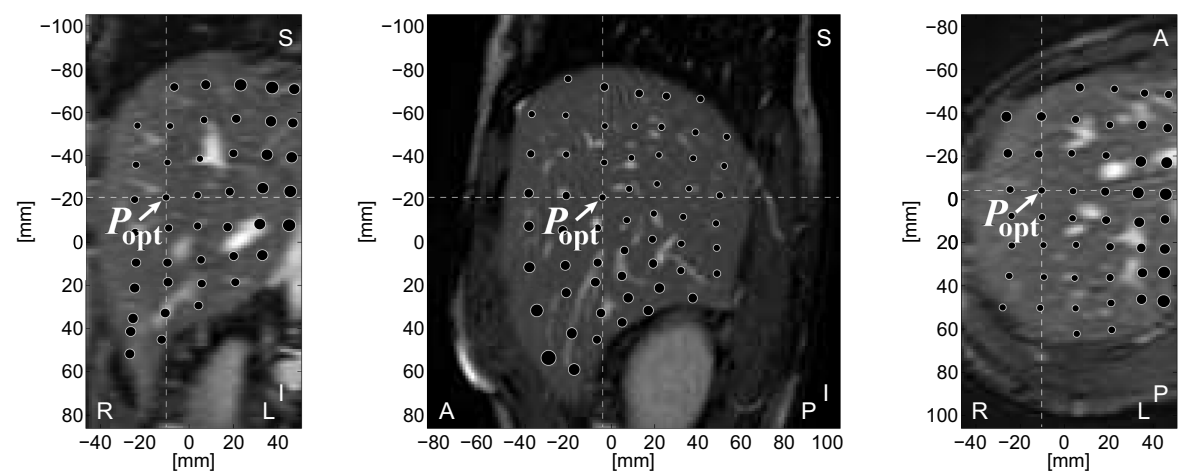

Fig. 3. Prediction errors when different points are used as predictors. The radius of the markers indicates the mean prediction error over the right liver lobe that results if this specific point can be measured. $P_{\mathrm{opt}}$ is the point with maximum potential for variance reduction according to the model.

prediction criterion, i.e. the Mahalanobis distance from the mean shape, takes the entire liver into account and may be not optimal for single points.

Case B: Table 1 further shows the prediction error if $P_{\text {opt }}$ cannot be localised but one of its six direct neighbours. The small degradations again show the robustness with respect to the exact choice of the predictor.

Case $C$ : To evaluate the further improvements in prediction accuracy with more available measurements, we applied (2) to find not only one but three points with maximum potential for variance reduction. In each leave-one-out model the triplet with maximum variance reduction was found by exhaustive search. These three points were then assumed to be measurable in the left-out liver and used as predictors. The quantitative results for three predictors in Table 1 show a clear reduction of the prediction error in all but one subject. Only subject 10 shows a slight increase from $1.4 \mathrm{~mm}$ to $1.5 \mathrm{~mm}$ in the maximum prediction error with 3 known points compared to the prediction based on $P_{\text {opt }}$. This is again possible, because the prediction criterion was defined over the entire liver lobe and may insufficiently reflect single point deviations. However, the mean prediction error of $0.5 \mathrm{~mm}$ in this subject is the smallest among all experiments.

\section{Discussion and Conclusions}

In the described experiments to evaluate the predictive power of the model we assumed accurately measurable predictors. An important further step will be to incorporate the uncertainty of measurements and to determine the achievable accuracy in practical setups. Moreover, the kind of partial information used for prediction may be adapted to a certain application. For example, if only the inplane components of the predictor displacement are measurable, then the model 
and the prediction framework are not changed, but more measurements may be necessary for accurate predictions.

The mechanically motivated correspondences between subjects led to a compact model of liver deformation. However, a more complex scheme, which takes more mechanically relevant landmarks into account, such as the contact areas with the right kidney, the gall bladder or the portal vein, may further increase the predictive power of the model.

The presented model was built to specifically capture the deformation of the liver in addition to its quasi-periodic motion. A model of the full respiratory motion covering the complete respiratory cycle, changes in amplitude, and variations of the breathing speed would further broaden the spectrum of applications and is the subject of ongoing research.

Acknowledgments. This work has been supported by the CO-ME/NCCR research network of the Swiss National Science Foundation. The authors would like to thank Urs Gamper and Prof. Dr. Peter Boesiger (Institute for Biomedical Engineering, ETH Zurich) for their support in image acquisition.

\section{References}

1. Davies, S.C., Hill, A.L., Holmes, R.B., et al.: Ultrasound quantitation of respiratory organ motion in the upper abdomen. Br. J. Radiol. 67, 1096-1102 (1994)

2. Mageras, G.S., Yorke, E., Rosenzweig, K., et al.: Fluoroscopic evaluation of diaphragmatic motion reduction with a respiratory gated radiotherapy system. J. App. Clin. Med. Phys. 2, 191-200 (2001)

3. von Siebenthal, M., Székely, G., Gamper, U., et al.: 4D MR imaging of respiratory organ motion and its variability. Phys. Med. Biol. 52(6), 1547-1564 (2007)

4. Davatzikos, C., Shen, D., Mohamed, A., Kyriacou, S K: A framework for predictive modeling of anatomical deformations. IEEE T. Med. Imaging 20, 836-843 (2001)

5. Blackall, J.M., Penney, G.P., King, A.P., Hawkes, D.J.: Alignment of sparse freehand 3-D ultrasound with preoperative images of the liver using models of respiratory motion and deformation. IEEE T. Med. Imaging 24, 1405-1416 (2005)

6. Perperidis, D., et al.: Construction of a 4D statistical atlas of the cardiac anatomy and its use in classification. In: Duncan, J.S., Gerig, G. (eds.) MICCAI 2005. LNCS, vol. 3750, pp. 402-410. Springer, Heidelberg (2005)

7. Rueckert, D., Sonoda, L I, et al.: Nonrigid registration using free-form deformations: application to breast MR images. IEEE T. Med. Imaging 18, 712-721 (1999)

8. Styner, M., et al.: Evaluation of 3D correspondence methods for model building. In: Taylor, C.J., Noble, J.A. (eds.) IPMI 2003. LNCS, vol. 2732, pp. 63-75. Springer, Heidelberg (2003)

9. Hug, J., Brechbühler, C., Székely, G.: Model-based initialisation for segmentation. In: Vernon, D. (ed.) ECCV 2000. LNCS, vol. 1843, pp. 290-306. Springer, Heidelberg (2000) 